\title{
Fuzzy Approach for Group Sequential Test
}

\author{
Duygu İçen, Sevil Bacanlı, and Süleyman Günay \\ Department of Statistics, Hacettepe University, 06800 Ankara, Turkey \\ Correspondence should be addressed to Duygu İçen; duyguicn@hacettepe.edu.tr
}

Received 10 November 2013; Revised 26 December 2013; Accepted 26 December 2013; Published 19 February 2014

Academic Editor: Ping Feng Pai

Copyright (C) 2014 Duygu İçen et al. This is an open access article distributed under the Creative Commons Attribution License, which permits unrestricted use, distribution, and reproduction in any medium, provided the original work is properly cited.

\begin{abstract}
Buckley's approach (Buckley (2004), (2005), (2006)) uses sets of confidence intervals by taking into consideration both of the uncertainty and impreciseness of concepts that produce triangular shaped fuzzy numbers for the estimator. This approach produces fuzzy test statistics and fuzzy critical values in hypothesis testing. In addition, the sample size is fixed for this test. When data comes sequentially, however, it is not suitable to study with a fixed sample size test. In such cases, sequential and group sequential tests are recommended. Unlike a sequential test, a group of sequential test provides substantial savings in sample and enables us to make decisions as early as possible. This intends paper to combine the benefits of group sequential test and Buckley's approach using $\alpha$-cuts. It attempts to show that using $\alpha$-cuts can be used within the group sequential tests. To illustrate the test more explicitly a numerical example is also given.
\end{abstract}

\section{Introduction}

Estimation of unknown parameters of statistical models or testing of statistical hypothesis in fuzzy environments are interesting subjects for different approaches. So far Zadeh [1], Taheri and Behboodian [2,3], Taheri [4], Torabi and Behboodian [5], and Taheri and Arefi [6] have worked on these issues. Using a different point of view, Buckley [7-9] developed an approach which uses a set of confidence intervals. In addition, many studies have been done to combine several statistical methods and fuzzy sets, called fuzzy statistics, such as regression analysis, time series analysis, design of experiments, probability theory, conjoint analysis, and control charts [10]. Since statistical tests based on fuzzy test statistics are more flexible than classical tests, they seemed to be competitive tools in certain situations; for example, when the observed value of the test statistic is close to the quantile of the test statistic [6].

Fixed sample size test is not useful where subjects enter the study sequentially. And thus the accumulated data can be analyzed sequentially. Wald [11] introduced a sequential probability ratio test (SPRT) which requires substantially fewer observations than a fixed sample size test. Several authors such as Torabi and Behboodian [12] have proposed fuzzy sequential probability ratio test. Talukdar and Baruah
[13] have fuzzified the SPRT. Torabi and Mirhosseini [14] introduced the SPRT for fuzzy hypotheses testing. Jamkhaneh and Gildeh [15] presented a new approach for SPRT based on fuzzy hypothesis.

SPRT has no finite maximum number of observation; it is generally considered inappropriate for clinical trials [16]. Group sequential tests (GST) are generally more practical and they give more possible savings than SPRT when it is impractical to perform an interim analysis after each new observation [17]. GST are widely used in clinical trials. For ethical, scientific, and economic reasons, clinical trials are often repeatedly monitored for evidence of treatment benefit or harm. To achieve this, statisticians conduct interm analysis periodically on accumulating data [18]. Various group sequential testing procedures have been proposed to achieve the desired levels of type-I error [18-20]. Much of the development of GST are reviewed in detail by Jennison and Turnbull [17].

None of the studies mentioned above consider Buckley's approach for group sequential tests [7-9]. In this study, our aim is to use a group sequential test using $\alpha$-cuts inspired by Buckley's approach when response variable has a normal distribution with known variance.

This paper is organized as follows: some preliminaries for fuzzy numbers are presented in Section 2. Buckley's approach 
using $\alpha$-cuts for hypothesis testing is briefly reviewed in Section 3. Then Pocock's group sequential test and using Buckley's approach within Pocock's group sequential test for a normal response with known variance are given in Section 4. A numerical example is given in Section 5. Results and discussions are given in Section 6. Finally, concluding remarks and some possible future perspectives are presented in Section 7 .

\section{Preliminaries}

This section contains some definitions of fuzzy sets and fuzzy numbers defined by Dubois and Prade [21] and Buckley [7-9].

Definition 1. A fuzzy number $\widetilde{A}$ is a fuzzy subset of the real line $\mathfrak{R}$. Its membership function $\mu_{A}(x)$ satisfies the following criteria [21]:

(i) $\alpha$-cut set of $\mu_{A}(x)$ is a closed interval,

(ii) $\exists x$ such that $\mu_{A}(x)=1$, and

(iii) convexity such that $\mu_{A}\left(\lambda x_{1}+(1-\lambda) x_{2}\right) \geq$ $\min \left(\mu_{A}\left(x_{1}\right), \mu_{A}\left(x_{2}\right)\right)$ for $\lambda \in[0,1]$,

where $\alpha$-cut set contains all $x$ elements that have a membership grade $\mu_{A}(x) \geq \alpha$.

Definition 2. A triangular shaped fuzzy number $\widetilde{A}(T S F N)$ is a fuzzy number, whose membership function is defined by three values, $a_{1}<a_{2}<a_{3}$, where the base of triangular is the interval $\left[a_{1}, a_{3}\right]$ and the vertex is $x=a_{2}$ [21].

Definition 3. The $\alpha$-cut of a fuzzy number $\widetilde{A}$ is a nonfuzzy set defined as $\widetilde{A}(\alpha)=\left\{x \in \mathfrak{R}, \mu_{A}(x) \geq \alpha\right\}$. Hence $\widetilde{A}(\alpha)=$ $\left[A^{L}(\alpha), A^{U}(\alpha)\right]$, where $A^{L}(\alpha)=\inf \left\{x \in \Re, \mu_{A}(x) \geq \alpha\right\}$ and $A^{U}(\alpha)=\sup \left\{x \in \Re, \mu_{A}(x) \geq \alpha\right\}[21]$.

Definition 4. Consider $X$ a random variable with probability density function $N\left(\mu, \sigma^{2}\right)$, which is the normal probability density with unknown mean $\mu$, a known variance $\sigma^{2}$. To estimate $\mu$, a random sample $X_{1}, \ldots, X_{n}$ from $N\left(\mu, \sigma^{2}\right)$ is obtained. Suppose that the mean of this random sample turns out to be $\bar{X}$, which is a crisp number. It is known that $\bar{X}$ is $N\left(\mu, \sigma^{2} / n\right)$; therefore, $(\bar{X}-\mu) /(\sigma / \sqrt{n})$ is $N(0,1)$. So

$$
P\left(-z_{\gamma / 2} \leq \frac{\bar{X}-\mu}{\sigma / \sqrt{n}} \leq z_{\gamma / 2}\right)=1-\gamma,
$$

where $z_{\gamma / 2}$ is the $z$ value that the probability of a $N(0,1)$ random variable exceeding it is $\gamma / 2$ ( $\gamma$ is the type-I error). Then inequality is solved to produce $\mu$ that is given as follows:

$$
P\left(\bar{X}-\frac{z_{\gamma / 2} \sigma}{\sqrt{n}} \leq \mu \leq \bar{X}+\frac{z_{\gamma / 2} \sigma}{\sqrt{n}}\right)=1-\gamma .
$$

This leads directly to the $(1-\gamma) 100 \%$ confidence interval for $\mu$

$$
\left[\theta_{1}(\gamma), \theta_{2}(\gamma)\right]=\left[\bar{X}-\frac{z_{\gamma / 2} \sigma}{\sqrt{n}}, \bar{X}+\frac{z_{\gamma / 2} \sigma}{\sqrt{n}}\right]
$$

where

$$
\int_{-\infty}^{z_{\gamma / 2}} N(0,1) d x=1-\frac{\gamma}{2}
$$

and $N(0,1)$ denotes the normal density with mean zero and unit variance. With putting these confidence intervals one on top of other, we obtain $\widetilde{X}$ that is the fuzzy estimator of $\mu$ [7-9] whose $\alpha$-cuts are confidence intervals as

$$
\widetilde{X}[\alpha]=\left[\bar{X}-\frac{z_{\alpha / 2} \sigma}{\sqrt{n}} ; \bar{X}+\frac{z_{\alpha / 2} \sigma}{\sqrt{n}}\right],
$$

for $0.01 \leq \alpha \leq 1$. Hence we obtain the fuzzy estimator of $\mu$.

\section{Hypothesis Testing Using $\alpha$-Cuts}

Buckley's approach [7-9] uses set of confidence intervals producing a triangular shaped fuzzy number for the estimator. Therefore this approach produces a fuzzy test statistic and fuzzy critical values in fuzzy hypothesis testing.

In this section the classical hypothesis test, based on fixed sample size of $n$ from $N\left(\mu, \sigma^{2}\right)$ mean and variance known, is given. The following hypothesis $H_{0}: \mu=\mu_{0}$ against $H_{1}$ : $\mu \neq \mu_{0}$ is going to be tested at significance level $\gamma$.

From the random sample its mean is computed as $\bar{X}$ and then the test statistic is determined as

$$
Z_{0}=\frac{\bar{X}-\mu_{0}}{\sigma / \sqrt{n}} .
$$

Let $\gamma, 0<\gamma<1$, denote the significance level of the test. Now under the null hypothesis $H_{0}, Z_{0}$ is $N(0,1)$ and decision rule is (1) reject $H_{0}$ if $Z_{0} \geq z_{\gamma / 2}$; (2) reject $H_{0}$ if $Z_{0} \leq-z_{\gamma / 2}$, and (3) do not reject $H_{0}$ when $-z_{\gamma / 2}<Z_{0}<z_{\gamma / 2}$. In the above decision rule $z_{\gamma / 2}$ is the $z$ value so that the probability of a random variable having the $N(0,1)$ probability density exceeding $z$ is $\gamma / 2$ ( $\gamma$ is the type-I error rate) [7].

If the uncertainty is taken into account for parameter $\mu$, triangular shaped fuzzy number $\widetilde{X}$ and its $\alpha$-cuts $(\alpha \in$ $[0.01,1])$ can be given with Definition 4 in (7)

$$
\widetilde{Z}=\frac{\widetilde{X}-\mu_{0}}{\sigma / \sqrt{n}}
$$

Calculations are performed by $\alpha$-cuts and interval arithmetic. Substituting the bounds of $\widetilde{X}[\alpha]$ into (7), all $\alpha$-cuts of $\widetilde{Z}$ can be given with (8)

$$
\begin{aligned}
\widetilde{Z}[\alpha] & =\frac{\widetilde{X}[\alpha]-\mu_{0}}{\sigma / \sqrt{n}} \\
& =\left[\frac{\bar{X}-z_{\alpha / 2} \sigma / \sqrt{n}-\mu_{0}}{\sigma / \sqrt{n}} ; \frac{\bar{X}+z_{\alpha / 2} \sigma / \sqrt{n}-\mu_{0}}{\sigma / \sqrt{n}}\right] \\
& =\left[\frac{\bar{X}-\mu_{0}}{\sigma / \sqrt{n}}-z_{\alpha / 2} ; \frac{\bar{X}-\mu_{0}}{\sigma / \sqrt{n}}+z_{\alpha / 2}\right] \\
& =\left[Z_{0}-z_{\alpha / 2} ; Z_{0}+z_{\alpha / 2}\right] .
\end{aligned}
$$




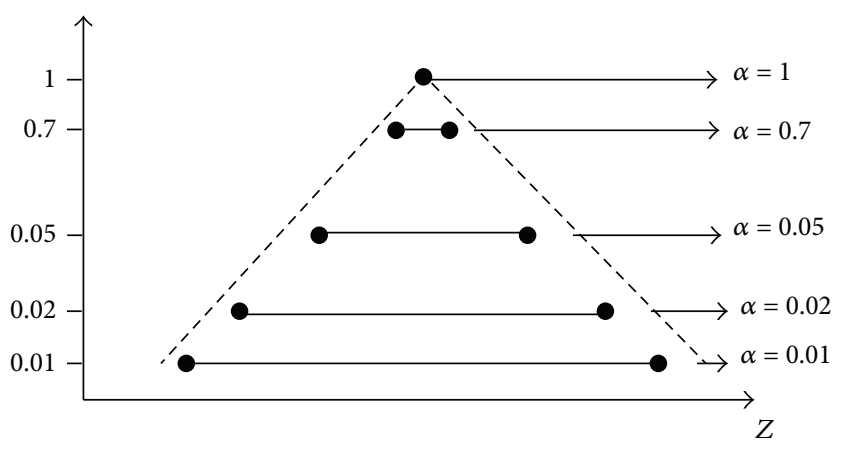

FIgURE 1: Fuzzy test statistic.

Each $\alpha$-cut is put one over the other in order to get a triangular fuzzy test statistic $\widetilde{Z}[\alpha]$ which is given in Figure 1 .

Since the test statistics are fuzzy the critical values will also be fuzzy. There will be two fuzzy critical value sets: (1) let $\widetilde{\mathrm{CV}}_{1}$ correspond to $-z_{\gamma / 2}$ and (2) let $\widetilde{\mathrm{CV}}_{2}$ go with $z_{\gamma / 2}$. Set $\widetilde{\mathrm{CV}}_{i}[\alpha]=$ $\left[\mathrm{cv}_{i 1}(\alpha), \mathrm{cv}_{i 2}(\alpha)\right], i=1,2$. The end points of an $\alpha$-cut of $\widetilde{\mathrm{CV}}_{2}$ are computed from the end points of the corresponding $\alpha$-cut of $\widetilde{Z}$ with the following equations:

$$
\operatorname{Prob}\left(Z_{0}+z_{\alpha / 2}>\mathrm{cv}_{22}\right)=\frac{\gamma}{2} \text {. }
$$

Hence under $H_{0}, Z_{0}$ is $N(0,1)$ so

$$
\mathrm{cv}_{22}(\alpha)=z_{\gamma / 2}+z_{\alpha / 2}
$$

By using the left end point of $\widetilde{Z}[\alpha]$ in (9), we have

$$
\mathrm{cv}_{21}(\alpha)=z_{\gamma / 2}-z_{\alpha / 2}
$$

Hence $\alpha$-cuts of $\widetilde{\mathrm{CV}}_{2}$ and $\widetilde{\mathrm{CV}}_{1}$ are given by

$$
\begin{aligned}
& \widetilde{\mathrm{CV}}_{2}[\alpha]=\left[z_{\gamma / 2}-z_{\alpha / 2}, z_{\gamma / 2}+z_{\alpha / 2}\right], \\
& \widetilde{\mathrm{CV}}_{1}[\alpha]=-\widetilde{\mathrm{CV}}_{2}[\alpha], \\
& \widetilde{\mathrm{CV}}_{1}[\alpha]=\left[-z_{\gamma / 2}-z_{\alpha / 2},-z_{\gamma / 2}+z_{\alpha / 2}\right],
\end{aligned}
$$

respectively $[7,8]$. Both $\widetilde{\mathrm{CV}}_{1}$ and $\widetilde{\mathrm{CV}}_{2}$ are triangular shaped fuzzy numbers. Since the crisp test statistics has a normal distribution, $\widetilde{\mathrm{CV}}_{1}=-\widetilde{\mathrm{CV}}_{2}$ because this density is symmetric with respect to zero [7-9]. The final decision depends on the relationship between $\widetilde{Z}$ and $\widetilde{C V}_{1}$ and $\widetilde{C V}_{2}$ : (1) $\widetilde{Z}>\widetilde{\mathrm{CV}}_{2}$ reject $H_{0}$ (Figure 2(a)); (2) $\widetilde{Z}<\widetilde{\mathrm{CV}}_{1}$ reject $H_{0}$ (Figure 2 (b)); (3) $\widetilde{\mathrm{CV}}_{1}<\widetilde{Z}<\widetilde{\mathrm{CV}}_{2}$ do not reject Ho (Figure 2(c)); and (4) $\widetilde{\mathrm{CV}}_{1} \approx \widetilde{Z}<\widetilde{\mathrm{CV}}_{2}$ or $\widetilde{\mathrm{CV}}_{1}<\widetilde{Z} \approx \widetilde{\mathrm{CV}}_{2}$ no decision (Figures $2(\mathrm{~d})$ and $2(\mathrm{e}))$. These situations are explained in detail as follows. For example, if $\widetilde{Z}>\widetilde{\mathrm{CV}}_{2}$, draw $\widetilde{Z}$ to the right of $\widetilde{\mathrm{CV}}_{2}$, then find the height of the intersection as

$$
v\left(\widetilde{Z} \leq \widetilde{\mathrm{CV}}_{2}\right)=\max \left\{\min \left(\widetilde{Z}(x), \widetilde{\mathrm{CV}}_{2}(y)\right) \mid x \leq y\right\},
$$

which measures how much $\widetilde{\mathrm{CV}}_{2}(y)$ is less than or equal to $\widetilde{Z}(x)$. Thus, $\widetilde{\mathrm{CV}}_{2}<\widetilde{Z}$ if $v\left(\widetilde{Z} \leq \widetilde{\mathrm{CV}}_{2}\right)<\eta$, where $\eta$ is some fixed fraction in $(0,1]$ (Figure 2(a)). Other situations are summarized in Figures 2(b), 2(c), 2(d), and 2(e). In this figure, the height of the intersection is $y_{0}$ and $\eta=0.8$ as Buckley [7-9] states. Now the results can be given as (1) if $y_{0}<0.8$, then $\widetilde{Z}>\widetilde{\mathrm{CV}}_{2}$ (Figure 2(a)) and (2) if $y_{0} \geq 0.8$ then $\widetilde{Z} \approx \widetilde{C V}_{i}, i=1,2$ (Figures 2 (d) and 2(e)). Similar results hold for $\widetilde{Z}$ versus $\widetilde{C V}_{1}$ (Figures 2(b), 2(c), 2(d), and 2(e)).

It is interesting that after evaluating $\widetilde{Z}$ and $\widetilde{\mathrm{CV}}_{1}, \widetilde{\mathrm{CV}}_{2}$, if $\widetilde{Z} \approx \widetilde{C V}_{i}, i=1,2$ (Figures $2(\mathrm{~d})$ and $2(\mathrm{e})$ ) then the final decision is "no decision" on $H_{0}$. This is because of the fuzzy numbers that incorporate all uncertainty in confidence intervals [7-9]. Consequently, hypothesis testing based on fuzzy test statistic and fuzzy critical values that is described above is more realistic and provides more benefits when value of the test statistic is very near to the quantile of the test statistic.

\section{Group Sequential Test Using $\alpha$-Cuts}

In Pocock's group sequential test, subject entry is divided into $K$ equally sized groups containing $n$ subject on each treatment and the data are analyzed after each new group [20]. Consider the response variables to be normal with unknown means $\mu_{1}$ and $\mu_{2}$ with a common variance $\sigma^{2}$. Subjects are randomized sequentially into two treatment groups.

The paper is planned as a test of the null hypothesis $H_{0}$ : $\mu_{1}=\mu_{2}$ against the two sided alternative $H_{1}: \mu_{1} \neq \mu_{2}$. Let $\bar{X}_{1 j}$ and $\bar{X}_{2 j}$ be the observed mean responses in the $j$ th group of $2 n$ subjects, then the statistics,

$$
Z_{j}=\frac{\sqrt{n}\left(\bar{X}_{1 j}-\bar{X}_{2 j}\right)}{\sqrt{2 \sigma^{2}}}
$$

are normally distributed with mean $\Delta$ and unit variance, where $\Delta=E\left(Z_{j}\right)=\sqrt{n}\left(\mu_{1}-\mu_{2}\right) / \sqrt{2 \sigma^{2}}$. Therefore test statistic is defined with $S_{k}=\sum_{j=1}^{k} Z_{j}$ and $k=1,2, \ldots, K$, where $S_{k}$, under $H_{0}, S_{k} \sim N(0, k)$, is a partial sum process of independent identically distributed (i.i.d.) standard normal random variables $Z_{k} \sim N(0,1), k=1,2, \ldots, K$. Under $H_{1}, S_{k}$ is again partial sum of i.i.d. normal random variables, $Z_{k} \sim$ $N(\Delta, 1), k=1,2, \ldots, K, S_{k} \sim N(\Delta, k)$. Critical values of 


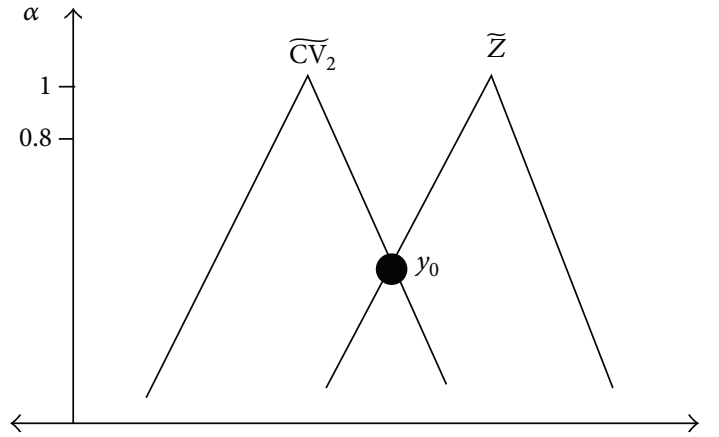

(a) Reject $H_{0}$ if $\widetilde{Z}>\widetilde{\mathrm{CV}}_{2}$

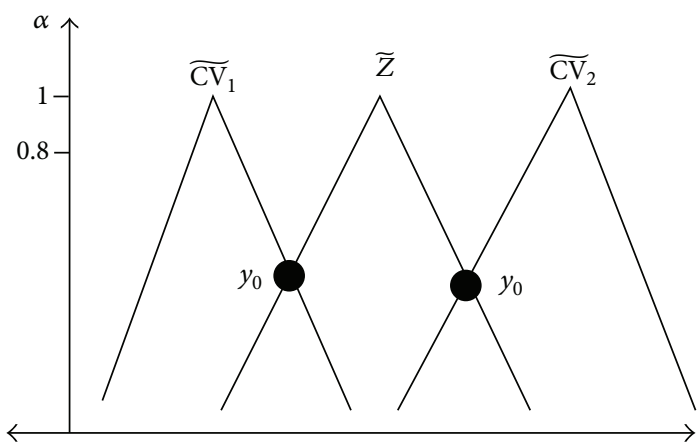

(c) Accept $H_{0}$ if $\widetilde{\mathrm{CV}}_{1}<\widetilde{Z}<\widetilde{\mathrm{CV}}_{2}$

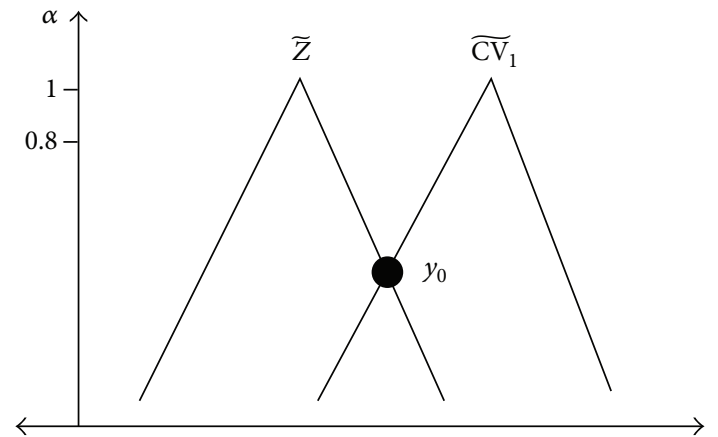

(b) Reject $H_{0}$ if $\widetilde{Z}<\widetilde{\mathrm{CV}}_{1}$

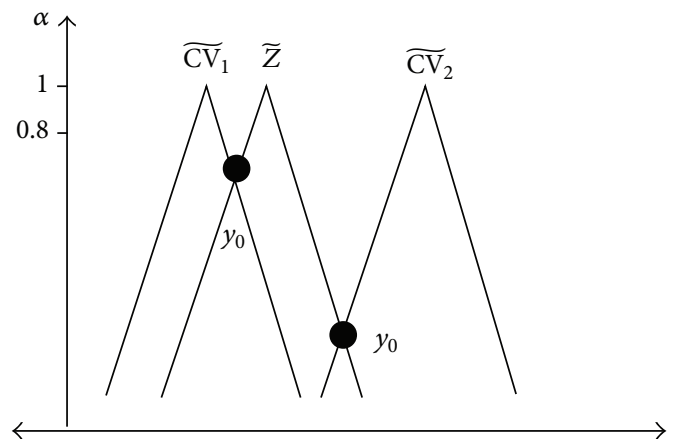

(d) No decision if $\widetilde{\mathrm{CV}}_{1} \approx \widetilde{Z}<\widetilde{\mathrm{CV}}_{2}$

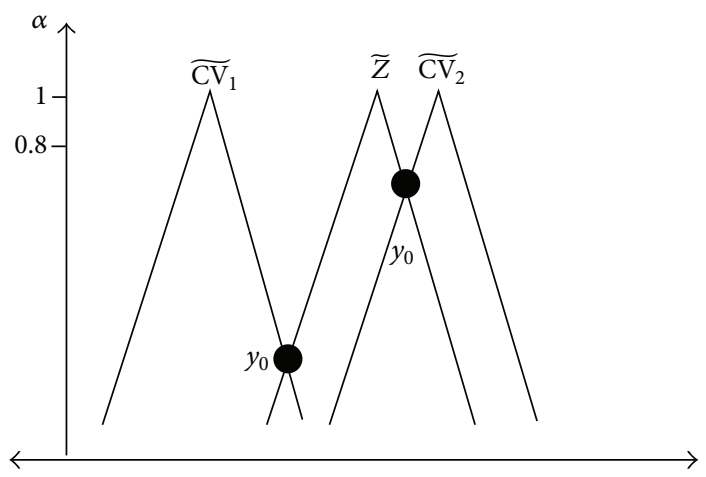

(e) No decision if $\widetilde{\mathrm{CV}}_{1}<\widetilde{Z} \approx \widetilde{\mathrm{CV}}_{2}$

FIgURE 2: Decision criteria for testing $H_{0}: \mu=\mu_{0}$ versus $H_{1}: \mu \neq \mu_{0}$ using $\alpha$-cuts.

Pococks's GST $\left(Z_{p}(K, \gamma)\right)$ are given in Table 1 for $K=1, \ldots, 5$; $1-\beta=0.95$, and $\gamma=0.10$. Then GST process is as follows:

(1) after group $k=1,2, \ldots, K-1$

if $\frac{\left|S_{k}\right|}{\sqrt{k}} \geq Z_{p}(K, \gamma), \quad$ stop, reject $H_{0}$,

otherwise, continue to group $k+1$,

(2) after group $K$

$$
\text { if } \frac{\left|S_{k}\right|}{\sqrt{K}} \geq Z_{p}(K, \gamma), \quad \text { stop, reject } H_{0},
$$

otherwise, stop, accept $H_{0}$.

(16)
TABLE 1: Pocock's critical values $Z_{p}(K, \gamma)$ and $\Delta$ for two-sided test, $K=1, \ldots, 5 ; 1-\beta=0.95 ; \gamma=0.10$.

\begin{tabular}{|c|c|c|c|c|c|c|}
\hline$K$ & & & $Z_{p}(K, \gamma)$ & & & $\Delta$ \\
\hline 1 & 1.645 & & & & & 3.290 \\
\hline 2 & 1.875 & 1.875 & & & & 2.445 \\
\hline 3 & 1.993 & 1.993 & 1.993 & & & 2.035 \\
\hline 4 & 2.067 & 2.067 & 2.067 & 2.067 & & 1.782 \\
\hline 5 & 2.122 & 2.122 & 2.122 & 2.122 & 2.122 & 1.605 \\
\hline
\end{tabular}

The value of noncentrality parameter, $\Delta$, can be determined to achieve a given value of $1-\beta$ [17]. Corresponding to a specific $\Delta$ under $H_{1}$ to be detected, the required sample 
size per treatment per stage, $n$, at given $\gamma$ and $1-\beta$ with a maximum of $K$ stages, is obtained as

$$
n=\Delta^{2}\left(\frac{\sqrt{2 \sigma^{2}}}{\mu_{1}-\mu_{2}}\right)^{2} .
$$

The maximum sample size, $n$, to find $\Delta$ for maximum of $K$ stages is simply

$$
n_{\text {max }}=2 n K \text {. }
$$

Group sequential tests can also be used in an experiment with only one treatment in which the response results are compared with a known standard. For a normal response with a known variance and $H_{0}$ hypothesis mean $\mu_{0}$, the critical values $Z_{k}$ and $\Delta$ become $\sqrt{n}\left(\mu_{1}-\mu_{0}\right) / \sigma[17,22]$. When $K=$ 1 , that is, for fixed sample size test, (17) becomes the classical hypothesis test for normal response.

The test statistic defined with (14) can also be given as follows:

$$
Z_{j}=\frac{\sqrt{n}\left(\bar{X}_{j}-\phi_{0}\right)}{\sqrt{\sigma^{2}}} .
$$

Suppose that $X_{i}=\left(X_{1 i}-X_{2 i}\right) / \sqrt{2} i=1,2, \ldots, n$ and $X_{i} \sim$ $N\left(\phi, \sigma^{2}\right)$, where $\phi=\left(\mu_{1}-\mu_{2}\right) / \sqrt{2}$ [23]. Therefore the mean of $j$ group of $n$ subject $\bar{X}_{j}$ can be defined as $\bar{X}_{j}=\left(\sum_{i=1}^{n} X_{i}\right) / n$. Now proceed to the fuzzy situation of $\widetilde{X}_{j}$ that is explained for each group of $n$ subject in (5) as triangular shaped fuzzy number is defined as

$$
\widetilde{X}_{j}[\alpha]=\left[\bar{X}_{j}-\frac{\sigma}{\sqrt{n}} z_{(\alpha / 2)} ; \bar{X}_{j}+\frac{\sigma}{\sqrt{n}} z_{(\alpha / 2)}\right] .
$$

Substituting the $\alpha$-cuts of $\widetilde{X}_{j}$ into (19) and using interval arithmetic $\alpha$-cuts of the fuzzy test statistics are obtained as follows:

$$
\begin{aligned}
\widetilde{Z}_{j}[\alpha] & =\frac{\widetilde{X}_{j}[\alpha]-\phi_{0}}{\sigma / \sqrt{n}} \\
& =\left[\frac{\overline{X_{j}}-(\sigma / \sqrt{n}) z_{\alpha / 2}-\phi_{o}}{\sigma / \sqrt{n}} ; \frac{\overline{X_{j}}+(\sigma / \sqrt{n}) z_{\alpha / 2}-\phi_{o}}{\sigma / \sqrt{n}}\right] \\
& =\left[\frac{\overline{X_{j}}-\phi_{0}}{\sigma / \sqrt{n}}-z_{\alpha / 2} ; \frac{\overline{X_{j}}-\phi_{0}}{\sigma / \sqrt{n}}+z_{\alpha / 2}\right] \\
& =\left[Z_{j}-z_{\alpha / 2} ; Z_{j}+z_{\alpha / 2}\right] .
\end{aligned}
$$

In the sense of this fuzzification fuzzy group sequential test statistic, $\widetilde{S}_{k}$ can be defined as

$$
\frac{\sum_{j=1}^{k} \widetilde{Z}_{j}[\alpha]}{\sqrt{k}}=\widetilde{S}_{k}[\alpha], \quad k=1,2, \ldots, K .
$$

The defined test statistic sample size fuzzy test statistic for $K=1$ which is given with (7). Afterwards, $\alpha$-cuts of Pococks's fuzzy critical value $\left(\widetilde{\mathrm{PCV}}_{i}, i=1,2\right)$

$$
\widetilde{\operatorname{PCV}}_{i}[\alpha]=\left[\operatorname{pcv}_{i 1}(\alpha), \operatorname{pcv}_{i 2}(\alpha)\right], \quad i=1,2
$$

can be calculated. The following equations include calculations for $\widetilde{\mathrm{PCV}}_{2}[\alpha]$ and $\widetilde{\mathrm{PCV}}_{1}[\alpha]$, respectively:

$$
\operatorname{Prob}\left[Z_{j}+z_{\alpha / 2}>\operatorname{pcv}_{22}(\alpha)\right]=\gamma \text {. }
$$

Hence

$$
\operatorname{pcv}_{22}(\alpha)=\left(Z_{p}(K, \gamma)+z_{\alpha / 2}\right)
$$

By using the left end point of $\widetilde{Z}_{j}[\alpha]$ given with (21) it is possible to have

$$
\operatorname{pcv}_{21}(\alpha)=\left(Z_{p}(K, \gamma)-z_{\alpha / 2}\right)
$$

As a result we obtain $\alpha$-cuts of $\widetilde{\mathrm{PCV}}_{2}[\alpha]$ with (27)

$$
\widetilde{\mathrm{PCV}}_{2}[\alpha]=\left[Z_{p}(K, \gamma)-z_{\alpha / 2}, Z_{p}(K, \gamma)+z_{\alpha / 2}\right] \text {. }
$$

In the above equation for $\widetilde{\mathrm{PCV}}_{2}, \gamma$ is fixed and $\alpha$ ranges in the interval $[0.01,1]$. Now $\widetilde{\mathrm{PCV}}_{1}=-\widetilde{\mathrm{PCV}}_{2}$ so

$$
\widetilde{\operatorname{PCV}}_{1}[\alpha]=\left[-Z_{p}(K, \gamma)-z_{\alpha / 2},-Z_{p}(K, \gamma)+z_{\alpha / 2}\right] \text {. }
$$

Both $\widetilde{\mathrm{PCV}}_{1}$ and $\widetilde{\mathrm{PCV}}_{2}$ are triangular shaped fuzzy numbers. The final decision depends on the relationship between $\widetilde{S}_{k}$ and $\widetilde{\mathrm{PCV}}_{1}$ and $\widetilde{\mathrm{PCV}}_{2}$. Therefore fuzzy group sequential test process is as follows:

(1) after group $k=1,2, \ldots, K-1$

$$
\text { if } \begin{aligned}
\widetilde{S}_{k}<\widetilde{\mathrm{PCV}}_{1} \text { or } \widetilde{S}_{k}>\widetilde{\mathrm{PCV}}_{2} ; & \text { stop reject } H_{0}, \\
\text { otherwise; } & \text { continue to } k+1,
\end{aligned}
$$

(2) after group $K$

$$
\begin{aligned}
& \text { if } \widetilde{S}_{k}<\widetilde{\mathrm{PCV}}_{1} \text { or } \widetilde{S}_{k}>\widetilde{\mathrm{PCV}}_{2} ; \quad \text { stop, reject } H_{0} \text {, } \\
& \text { if } \widetilde{\mathrm{PCV}}_{1}<\widetilde{S}_{k}<\widetilde{\mathrm{PCV}}_{2} ; \quad \text { accept } H_{0} \text {, } \\
& \text { if } \widetilde{S}_{k} \approx \widetilde{\mathrm{PCV}}_{i} \text { for } i=1,2 \text {; no decision. }
\end{aligned}
$$

These situations are explained in detail in Figure 3. The final decision depends on the relationship between $\widetilde{S}_{k}$ and $\widetilde{\mathrm{PCV}}_{1}$ and $\widetilde{\mathrm{PCV}}_{2}$ : (1) $\widetilde{S}_{k}>\widetilde{\mathrm{PCV}}_{2}$ reject $H_{0}$ (Figure $3(\mathrm{a})$ ); (2) $\widetilde{S}_{k}<\widetilde{\mathrm{PCV}}_{1}$ reject $H_{0}$ (Figure 3(b)); (3) $\widetilde{\mathrm{PCV}}_{1}<\widetilde{S}_{k}<$ $\widetilde{\mathrm{PCV}}_{2}$ do not reject $H_{0}$ (Figure 3(c)); and (4) $\widetilde{\mathrm{PCV}}_{1} \approx \widetilde{S}_{k}<$ $\widetilde{\mathrm{PCV}}_{2}$ or $\widetilde{\mathrm{PCV}}_{1}<\widetilde{S}_{k} \approx \widetilde{\mathrm{PCV}}_{2}$ no decision (Figures $3(\mathrm{~d})$ and 3(e)). We take the height of the intersection $\eta=0.8$, which measures how much $\widetilde{\mathrm{PCV}}_{i}, i=1,2$ is less than, bigger than, or equal to $\widetilde{S}_{k}$. The advantage of $\alpha$-cuts approach to GST is that, instead of generating and processing a single confidence interval, all the confidence intervals at the same time are calculated in the process of corresponding fuzzy test statistic. Therefore in this study we showed that this advantage is also valid for the process of group sequential tests using $\alpha$-cuts. 


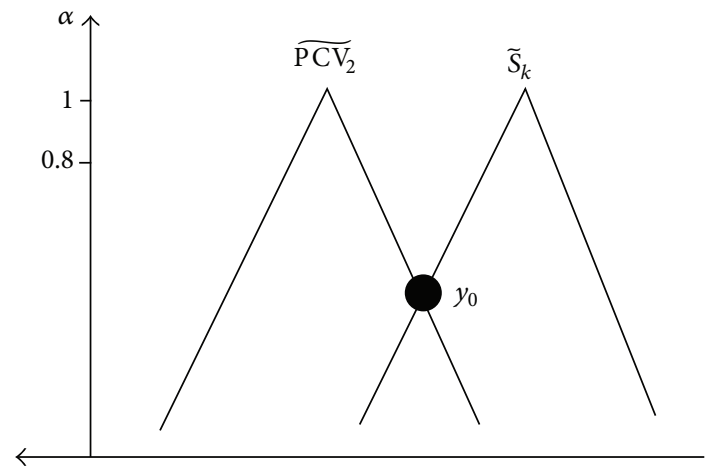

(a) Reject $H_{0}$ if $\widetilde{S}_{k}>\widetilde{\mathrm{PCV}}_{2}$

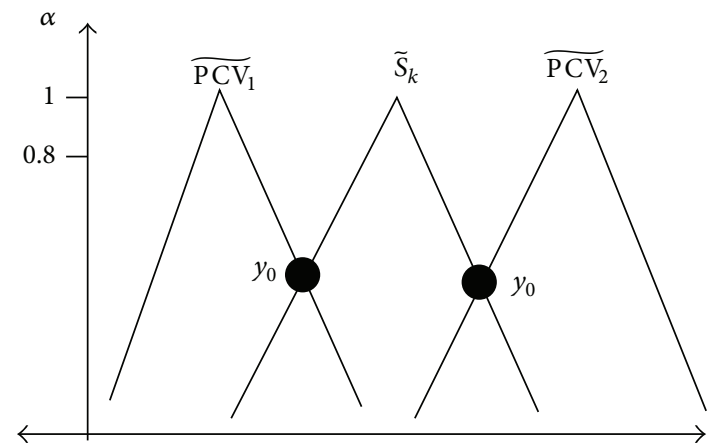

(c) Accept $H_{0}$ if $\widetilde{\mathrm{PCV}}_{1}<\widetilde{S}_{k}<\widetilde{\mathrm{PCV}}_{2}$

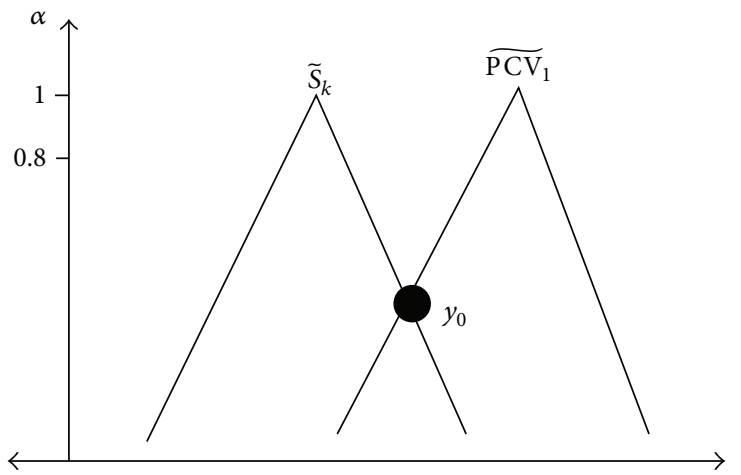

(b) Reject $H_{0}$ if $\widetilde{S}_{k}<\widetilde{\mathrm{PCV}}_{1}$

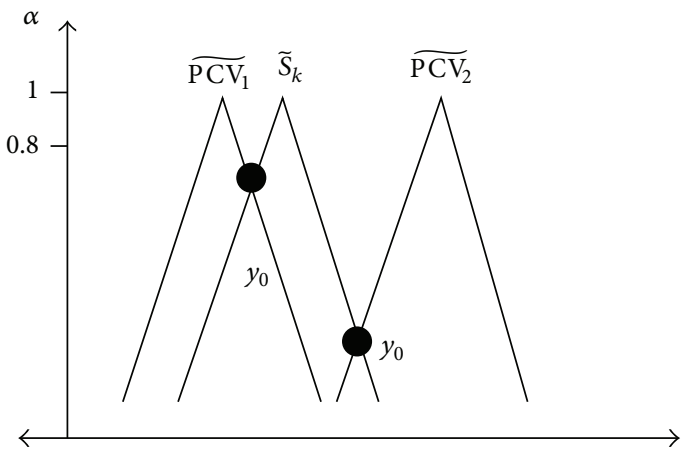

(d) No decision if $\widetilde{\mathrm{PCV}}_{1} \approx \widetilde{S}_{k}<\widetilde{\mathrm{PCV}}_{2}$

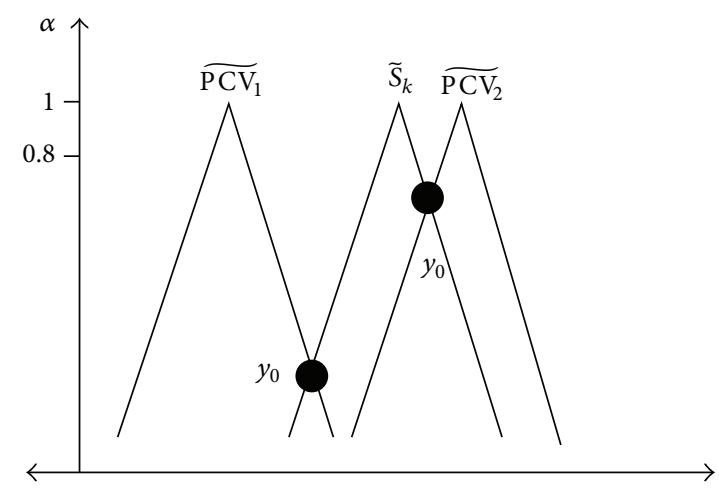

(e) No decision if $\widetilde{\mathrm{PCV}}_{1}<\widetilde{S}_{k} \approx \widetilde{\mathrm{PCV}}_{2}$

FIGURE 3: Decision criteria for $\alpha$-cuts approach to group sequential testing $H_{0}: \mu=\mu_{0}$ versus $H_{1}: \mu \neq \mu_{0}$.

\section{Numerical Example}

As an illustration we consider a real data in McCleve and Sincich [24] on page 381 given with Table 2 . The content of data is as follows: a new developed diet, that is, low in fats, carbonhydrates, and cholesterol, is intended to be used by people with heart disease. Furthermore the dietitian wishes to examine the effect that this diet has on the weights of obese people. Hence this data set concerns two groups which are called low fat diet (A) and regular diet (B), respectively.

We want to test $H_{0}: \mu_{\mathrm{A}}-\mu_{\mathrm{B}}=0$ with $\beta=\gamma=0.10, \sigma^{2}=$ 50.063, and $K=3$ at $H_{1}: \mu_{\mathrm{A}}-\mu_{\mathrm{B}}= \pm 5$; sample size which is calculated from (17) is $n_{\mathrm{A}}=n_{\mathrm{B}}=17$ per treatment per stage with maximum sample size $\left(n_{\max }\right)=102$ [23]. The fuzzy group sequential test statistics $\widetilde{S}_{1}, \widetilde{S}_{2}$, and $\widetilde{S}_{3}$ and fuzzy Pocock's critical values $\overparen{\mathrm{PCV}_{i}}, i=1,2,3$ are given in Table 3 .

The test statistic and critical value that is calculated in each stage is recalculated using Buckley's approach. Hence each $\alpha$-cut value is obtained for test statistic and critical value (for $\alpha=0.01,0.2,0.4,0.6,0.8$, and 1.00). $\alpha$-cuts, base and peak values of $\widetilde{S}_{1}, \widetilde{S}_{2}$, and $\widetilde{S}_{3}$, and critical values that are calculated in each stage are detailed in Table 4 . Thus, as suggested in Buckley's approach, not only one value but also more than one confidence interval is used, in order to test hypothesis, so that more information is included in group sequential test process. The results are obtained using the Maple 9 [25]. 
TABLE 2: Diet study data.

\begin{tabular}{|c|c|c|c|c|c|c|c|c|c|c|c|c|}
\hline & \multicolumn{4}{|c|}{$K=1$} & \multicolumn{4}{|c|}{$K=2$} & \multicolumn{4}{|c|}{$K=3$} \\
\hline \multirow{5}{*}{ (A) Low Fat Diet } & (1) 8 & (6) 3 & (11) 21 & (16) 20 & (1) 11 & (6) 10 & (11) 13 & (16) 8 & (1) 11 & (6) 4 & (11) 11 & (16) 2 \\
\hline & (2) 10 & (7) 11 & (12) 8 & (17) 14 & (2) 15 & (7) 12 & (12) 14 & (17) 10 & (2) 19 & (7) 11 & (12) 12 & (17) 4 \\
\hline & (3) 10 & (8) 7 & (13) 9 & & (3) 6 & (8) 1 & (13) 4 & & (3) 0 & (8) 7 & (13) 4 & \\
\hline & (4) 12 & (9) 9 & (14) 2 & & (4) 13 & (9) 7 & (14) 8 & & (4) 9 & (9) 14 & (14) 12 & \\
\hline & (5) 9 & (10) 2 & (15) 2 & & (5) 8 & (10) 10 & (15) 12 & & (5) 10 & (10) 2 & (15) 9 & \\
\hline \multirow{5}{*}{ (B) Regular Diet } & (1) 6 & (6) 6 & (11) 14 & (16) 8 & (1) 13 & (6) 6 & (11) 5 & (16) 6 & (1) 5 & (6) 8 & (11) 12 & (16) 13 \\
\hline & (2) 6 & (7) 10 & (12) 4 & (17) 8 & (2) 9 & (7) 11 & (12) 8 & (17) 8 & (2) 7 & (7) 13 & (12) 10 & (17) 11 \\
\hline & (3) 5 & (8) 3 & (13) 10 & & (3) 3 & (8) 12 & (13) 7 & & (3) 16 & (8) 1 & (13) 6 & \\
\hline & (4) 5 & (9) 9 & (14) 13 & & (4) 4 & (9) 9 & (14) 6 & & (4) 18 & (9) 9 & (14) 1 & \\
\hline & (5) 2 & (10) 11 & (15) 3 & & (5) 12 & (10) 8 & (15) 2 & & (5) 6 & (10) 8 & (15) 0 & \\
\hline
\end{tabular}

TABLE 3: Summarized calculations for fuzzy group sequential test.

\begin{tabular}{lcc}
\hline Step No & Test statistics & Critical values \\
\hline$\widetilde{Z}_{1}[\alpha]=\frac{\widetilde{X}_{1}-0}{\sigma / \sqrt{n}}=\left[1.26488-z_{(\alpha / 2)} ; 1.26488+z_{(\alpha / 2)}\right]$ & $\widetilde{\mathrm{PCV}}_{1}[\alpha]=\left[1.993-z_{(\alpha / 2)} ; 1.993-z_{(\alpha / 2)}\right]$ \\
& $\widetilde{S}_{1}[\alpha]=\left[1.26488-z_{(\alpha / 2)} ; 1.26488+z_{(\alpha / 2)}\right]$ & \\
$\widetilde{Z}_{2}[\alpha]=\frac{\widetilde{X}_{2}-0}{\sigma / \sqrt{n}}=\left[1.22768-z_{(\alpha / 2)} ; 1.22768+z_{(\alpha / 2)}\right]$ & $\widetilde{\mathrm{PCV}}_{2}[\alpha]=\left[1.993-z_{(\alpha / 2)} ; 1.993-z_{(\alpha / 2)}\right]$ \\
\hline$(2)$ & $\widetilde{S}_{2}[\alpha]=\frac{\left|\sum_{j=1}^{2} \widetilde{Z}_{j}\right|}{\sqrt{2}}=\frac{\left|\widetilde{Z}_{1}[\alpha]+\widetilde{Z}_{2}[\alpha]\right|}{\sqrt{2}}=\left[1.7625-z_{(\alpha / 2)} ; 1.7625+z_{(\alpha / 2)}\right]$ & \\
$\widetilde{Z}_{3}[\alpha]=\frac{\widetilde{X}_{3}-0}{\sigma / \sqrt{n}}=\left[1.533-z_{(\alpha / 2)} ; 1.533+z_{(\alpha / 2)}\right]$ & $\widetilde{\mathrm{PCV}}_{3}[\alpha]=\left[1.993-z_{(\alpha / 2)} ; 1.993-z_{(\alpha / 2)}\right]$ \\
& $\widetilde{S}_{3}[\alpha]=\frac{\left|\sum_{j=1}^{3} \widetilde{Z}_{j}\right|}{\sqrt{3}}=\frac{\left|\widetilde{Z}_{1}[\alpha]+\widetilde{Z}_{2}[\alpha]+\widetilde{Z}_{3}[\alpha]\right|}{\sqrt{3}}=\left[2.3248-z_{(\alpha / 2)} ; 2.3248+z_{(\alpha / 2)}\right]$ & \\
\hline
\end{tabular}

TABLE 4: Base and peak values $\left\{\left[a_{1}, a_{2}, a_{3}\right]\right\}$ of triangular shaped fuzzy numbers for group sequential test using $\alpha$-cuts for $K=3$ and $k=1,2,3$.

\begin{tabular}{|c|c|c|c|c|}
\hline \multirow{2}{*}{$\alpha$-cut } & \multicolumn{4}{|c|}{$k=1$} \\
\hline & $\widetilde{Z}_{1}$ & $\widetilde{S}_{1}$ & $\widetilde{\mathrm{PCV}}_{1}$ & $\widetilde{\mathrm{PCV}}_{2}$ \\
\hline 0.01 & {$[-1.23,1.265,3.761]$} & {$[-1.23,1.265,3.761]$} & {$[0.551,-1.993,-4.553]$} & {$[-0.551,1.993,4,553]$} \\
\hline 0.20 & {$[0.013,1.265,2.561]$} & {$[0.013,1.265,2.561]$} & {$[-0.714,-1.993,-3.271]$} & {$[0.714,1.993,3.271]$} \\
\hline 0.40 & {$[0.432,1.265,2.091]$} & {$[0.432,1.265,2.091]$} & {$[-1.113,-1.993,-2.831]$} & {$[1.113,1.993,2.831]$} \\
\hline 0.60 & {$[0.724,1.265,1.887]$} & {$[0.724,1.265,1.887]$} & {$[-1.487,-1.993,-2.534]$} & {$[1.487,1.993,2.534]$} \\
\hline 0.80 & {$[1.102,1.265,1.517]$} & {$[1.102,1.265,1.517]$} & {$[-1.763,-1.993,-2.266]$} & {$[1.763,1.993,2.266]$} \\
\hline 1.00 & {$[1.265,1.265,1.265]$} & {$[1.265,1.265,1.265]$} & {$[-1.993,-1.993,-1.993]$} & {$[1.993,1.993,1.993]$} \\
\hline \multirow{2}{*}{$\alpha$-cut } & \multicolumn{4}{|c|}{$k=2$} \\
\hline & $\widetilde{Z}_{2}$ & $\widetilde{S}_{2}$ & $\widetilde{\mathrm{PCV}}_{1}$ & $\widetilde{\mathrm{PCV}}_{2}$ \\
\hline 0.01 & {$[-1.331,1.235,3.782]$} & {$[-0.824,1.763,4.335]$} & {$[0.551,-1.993,-4.553]$} & {$[-0.551,1.993,4,553]$} \\
\hline 0.20 & {$[-0.074,1.235,2.513]$} & {$[0.468,1.763,3.023]$} & {$[-0.714,-1.993,-3.271]$} & {$[0.714,1.993,3.271]$} \\
\hline 0.40 & {$[0.394,1.235,2.122]$} & {$[0.967,1.763,2.645]$} & {$[-1.113,-1.993,-2.831]$} & {$[1.113,1.993,2.831]$} \\
\hline 0.60 & {$[0.756,1.235,1.693]$} & {$[1.253,1.763,2.284]$} & {$[-1.487,-1.993,-2.534]$} & {$[1.487,1.993,2.534]$} \\
\hline 0.80 & {$[0.997,1.235,1.486]$} & {$[1.511,1.763,1.987]$} & {$[-1.763,-1.993,-2.266]$} & {$[1.763,1.993,2.266]$} \\
\hline 1.00 & {$[1.235,1.235,1.235]$} & {$[1.763,1.763,1.763]$} & {$[-1.993,-1.993,-1.993]$} & {$[1.993,1.993,1.993]$} \\
\hline \multirow{2}{*}{$\alpha$-cut } & \multicolumn{4}{|c|}{$k=3$} \\
\hline & $\widetilde{Z}_{3}$ & $\widetilde{S}_{3}$ & $\widetilde{\mathrm{PCV}}_{1}$ & $\widetilde{\mathrm{PCV}}_{2}$ \\
\hline 0.01 & {$[-1.032,1.533,4.122]$} & {$[-0.274,2.325,4.923]$} & {$[0.551,-1.993,-4.553]$} & {$[-0.551,1.993,4,553]$} \\
\hline 0.20 & {$[0.278,1.533,2.775]$} & {$[1.078,2.325,3.587]$} & {$[-0.714,-1.993,-3.271]$} & {$[0.714,1.993,3.271]$} \\
\hline 0.40 & {$[0.684,1.533,2.356]$} & {$[1.458,2.325,3.184]$} & {$[-1.113,-1.993,-2.831]$} & {$[1.113,1.993,2.831]$} \\
\hline 0.60 & {$[1.084,1.533,2.023]$} & {$[1.836,2.325,2.835]$} & {$[-1.487,-1.993,-2.534]$} & {$[1.487,1.993,2.534]$} \\
\hline 0.80 & {$[1.321,1.533,1.786]$} & {$[2.079,2.325,2.586]$} & {$[-1.763,-1.993,-2.266]$} & {$[1.763,1.993,2.266]$} \\
\hline 1.00 & {$[1.533,1.533,1.533]$} & {$[2.325,2.325,2.325]$} & {$[-1.993,-1.993,-1.993]$} & {$[1.993,1.993,1.993]$} \\
\hline
\end{tabular}




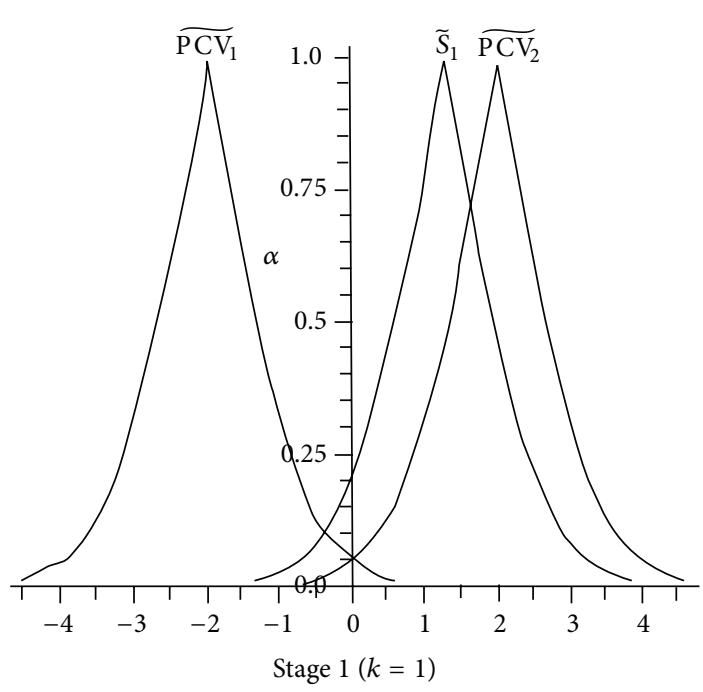

(a)

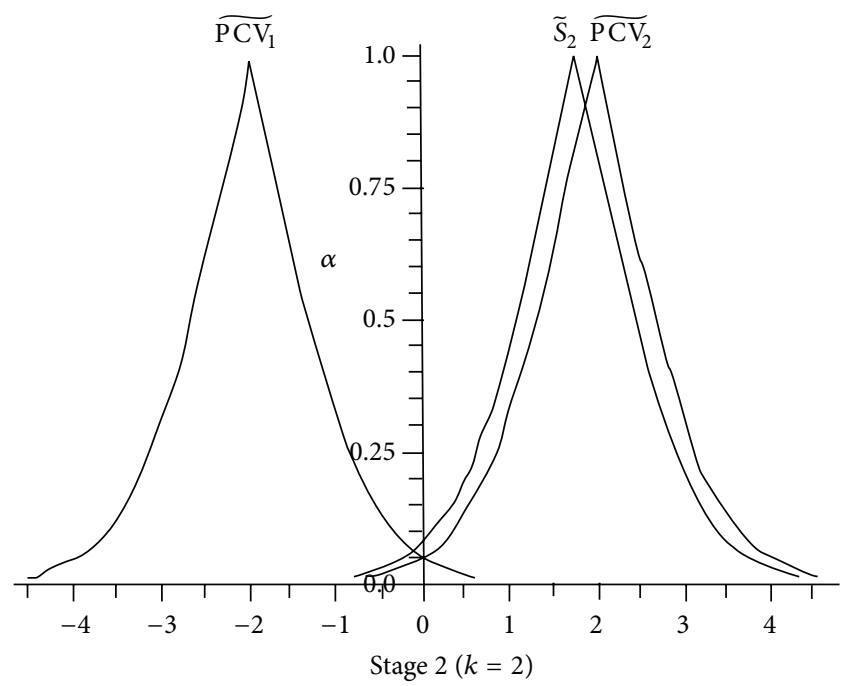

(b)

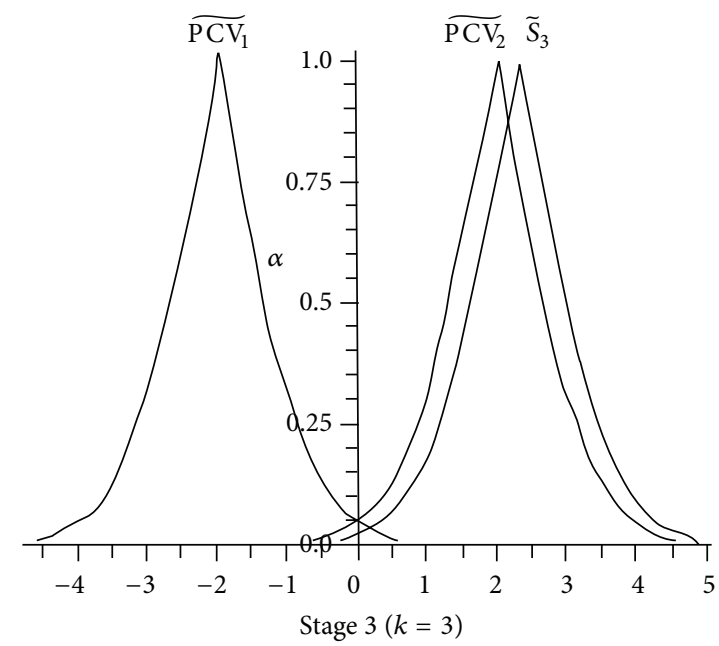

(c)

Figure 4: Summarized calculations for group sequential test using $\alpha$-cuts for $K=3, k=1,2,3$.

The graphical illustration of Table 4, which includes fuzzy group sequential test statistics and fuzzy critical values in each stage, is presented in Figure 4.

When Figure 4 is examined, $\alpha$-cut approach proposed by Buckley to group sequential test is seen more clearly. We take $\eta=0,8$ to sort the triangular shaped fuzzy numbers which are calculated by confidence intervals. This value is used in Buckley's work [7-9]. It is possible to take different $\eta$ values to test hypothesis. Thus, the flexibility of statistical tests based on fuzzy test statistics is said to be used for group sequential tests.

It is clear that $\widetilde{\mathrm{PCV}}_{1}<\widetilde{S}_{1}<\widetilde{\mathrm{PCV}}_{2}$ for first stage $(k=1)$ in Figure 4 . In this case we proceed to the second stage $(k=2)$. It is $\widetilde{S}_{2} \approx \widetilde{\mathrm{PCV}}_{2}$ in stage 2 , because the height of the intersection of two triangular shaped fuzzy numbers is very close to the value $\eta=0,8$. Therefore we proceed to the third stage $(k=3)$. When $k=3$ is examined, it is seen that $\widetilde{S}_{3}>\widetilde{\mathrm{PCV}}_{3}$ beacuse $\eta \approx 0,74$. As a result, $H_{0}$ is rejected. It is possible to say that the mean of two groups is different from each other.

\section{Results and Discussion}

In Buckley's approach, fuzzy test statistic is obtained by using more than one confidence interval as the $\alpha$-cut of triangular shaped fuzzy number. Thus, more information is used in hypothesis testing procedure. However sample size is fixed in the approach proposed by Buckley. Fixed sample size has no benefit in the studies where data comes sequentially. For this purpose, we show in this study how to use $\alpha$-cut approach proposed by Buckley in group sequential test. $\alpha$-cuts of the fuzzy test statistics and fuzzy critical values for each stage are calculated with (21) and (22) which are given in Section 4.

We apply Buckley's approcah to GST in an example given in Section 5. In this example if we take $\alpha=1$ at each stage, the fuzzy group sequential test returns into the classical group sequential test. This situation is given in Table 4 with $\alpha=1$. In classical group sequential testing procedure $S_{1}=1,265$ (for $k=1$ ), $S_{2}=1,763$ (for $k=2$ ), and $S_{3}=2,325$ (for $k=3$ ) are obtained. But is seems that especially in the second stage 
critical value and test statistics are very close to each other. Compared with each stage based on the critical value and test statistic, $H_{0}$ is rejected in third stage. In this situation only one value is considered in order to decide.

If we worked with fixed sample size in the example which is considered and if hypothesis was tested in accordance with Buckley's approach only, we would need 88 observations $\left(n_{\mathrm{A}}=44\right.$ and $\left.n_{\mathrm{B}}=44\right)$ for $K=1$ in (17). However, we started testing hypothesis with 34 observations $\left(n_{\mathrm{A}}=17\right.$ and $n_{\mathrm{B}}=$ 17 ) in total by using the $\alpha$-cut approach proposed by Buckley in the group sequential test for $K=3$.

\section{Conclusion}

In this study, we combine the benefits of $\alpha$-cut approach proposed by Buckley for the hypothesis testing procedure and GST. Fuzzy test statistics and fuzzy critical values proposed by Buckley are calculated for each step in the process of group sequential test and we achieved results that are more flexible and closer to the real life at the end of our study. In addition, group sequential test is done by considering more than one $\alpha$-cut value instead of only one $\alpha$ value $(\alpha=1)$, which is the advantage of fuzzy approach. Ultimately hypothesis is rejected. This situation is consistent with the classical situation. Consequently, it is shown that Buckley's approach can be used for Pocock's group sequential test as well. It is shown that the advantages of the two tests can be combined and used together in the application that is presented in this study.

\section{Conflict of Interests}

The authors declare that there is no conflict of interests regarding the publication of this paper.

\section{References}

[1] L. A. Zadeh, "Fuzzy sets," Information and Control, vol. 8, no. 3, pp. 338-353, 1965.

[2] S. M. Taheri and J. Behboodian, "Neyman-Pearson lemma for fuzzy hypotheses testing," Metrika, vol. 49, no. 1, pp. 3-17, 1999.

[3] S. M. Taheri and J. Behboodian, "A Bayesian approach to fuzzy hypotheses testing," Fuzzy Sets and Systems, vol. 123, no. 1, pp. 39-48, 2001.

[4] S. M. Taheri, "Trends in fuzzy statistics," Austrian Journal of Statistics, vol. 32, no. 3, pp. 239-257, 2003.

[5] H. Torabi and J. Behboodian, "Likelihood ratio tests for fuzzy hypotheses testing," Statistical Papers, vol. 48, no. 3, pp. 509$522,2007$.

[6] S. M. Taheri and M. Arefi, "Testing fuzzy hypotheses based on fuzzy test statistic," Soft Computing, vol. 13, no. 6, pp. 617-625, 2009.

[7] J. J. Buckley, Fuzzy Statistic, Springer, Hiedelberg, Germany, 2004.

[8] J. J. Buckley, "Fuzzy statistics: hypothesis testing," Soft Computing, vol. 9, no. 7, pp. 512-518, 2005.

[9] J. J. Buckley, Fuzzy Probability and Statistics, Springer, Berlin, Germany, 2006.
[10] S. Şentürk, "Fuzzy regression control chart based on $\alpha$-cut approximation," International Journal of Computational Intelligence Systems, vol. 3, no. 1, pp. 123-140, 2010.

[11] A. Wald, Sequential Analysis, John Wiley and Sons, New York, NY, USA, 1947.

[12] H. Torabi and J. Behboodian, "Sequential probability ratio test for fuzzy hypotheses testing with vague data," Austrian Journal of Statistics, vol. 34, no. 1, pp. 25-38, 2005.

[13] R. Talukdar and H. K. Baruah, "Sequential probability ratio test with fuzzy observations," The Journal of Fuzzy Mathematics, vol. 15, no. 3, pp. 627-643, 2007.

[14] H. Torabi and S. M. Mirhosseini, "Sequential probability ratio tests for fuzzy hypotheses testing," Applied Mathematical Sciences, vol. 3, no. 33-36, pp. 1609-1618, 2009.

[15] E. B. Jamkhaneh and B. S. Gildeh, "Sequential sampling plan using fuzzy SPRT," Journal of Intelligent and Fuzzy Systems, vol. 25, pp. 785-791, 2013.

[16] S. J. Pocock, "Interim analyses for randomized clinical trials: the group sequential approach," Biometrics, vol. 38, no. 1, pp. 153$162,1982$.

[17] C. Jennison and B. W. Turnbull, Sequential Methods with Applications to Clinical Trials, Chapman and Hall, New York, NY, USA, 2000.

[18] K. K. Gordon Lan and D. L. Demets, "Discrete sequential boundaries for clinical trials," Biometrika, vol. 70, no. 3, pp. 659663, 1983.

[19] P. C. O'Brien and T. R. Fleming, "A multiple testing procedure for clinical trials," Biometrics, vol. 35, no. 3, pp. 549-556, 1979.

[20] S. J. Pocock, "Group sequential methods in the design and analysis of clinical trials," Biometrika, vol. 64, no. 2, pp. 191-199, 1977.

[21] D. Dubois and H. Prade, "Operations with fuzzy numbers," International Journal of Systems Science, vol. 9, no. 6, pp. 613626, 1978.

[22] S. Bacanli and Y. P. Demirhan, "A group sequential test for the inverse Gaussian mean," Statistical Papers, vol. 49, no. 2, pp. 377$386,2008$.

[23] C. Jennison and B. W. Turnbull, "Repeated confidence intervals for group sequential clinical trials," Controlled Clinical Trials, vol. 5, no. 1, pp. 33-45, 1984.

[24] J. T. McCleve and T. Sincich, Statistics, Prentice Hall, Upper Saddle River, NJ, USA, 9th edition, 2003.

[25] “Maple 9," Waterloo Maple Inc, Waterloo, Canada, 2003. 

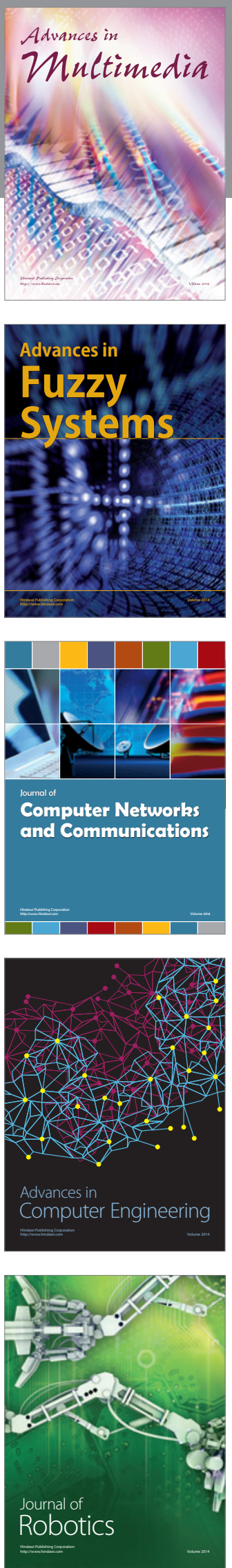

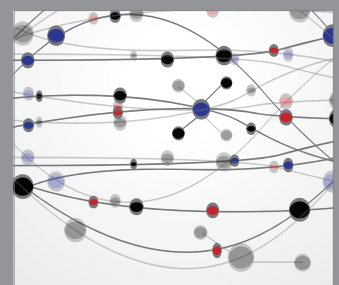

The Scientific World Journal
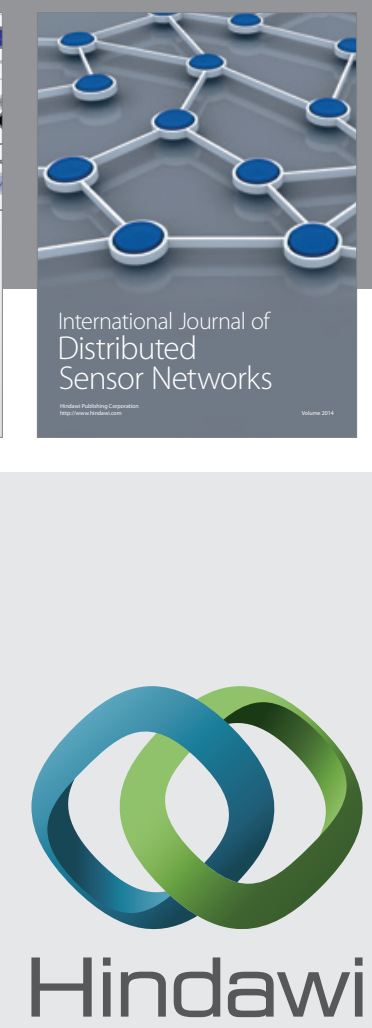

Submit your manuscripts at

http://www.hindawi.com
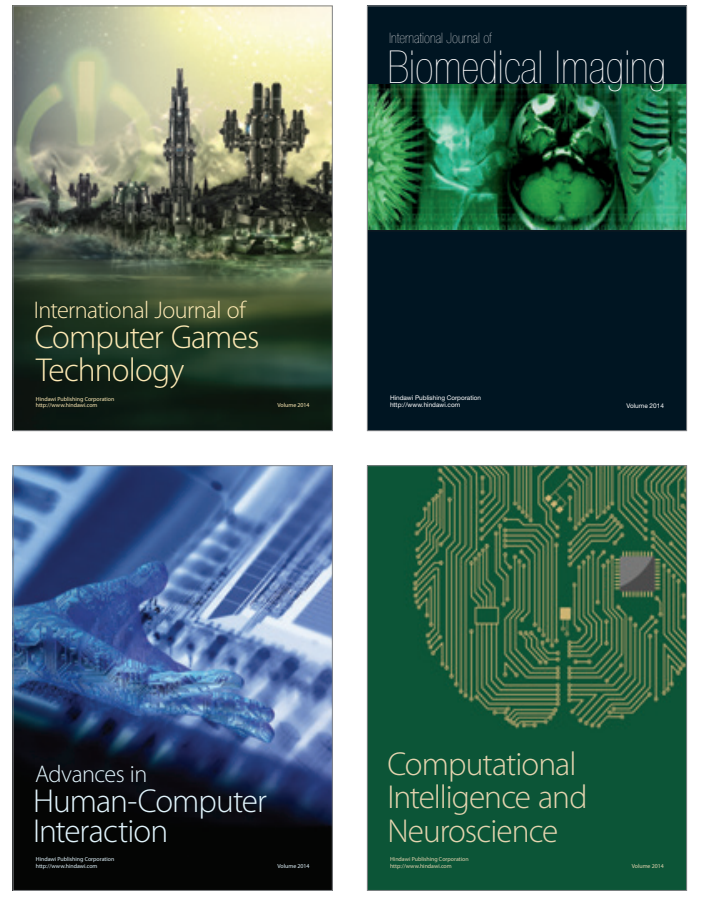
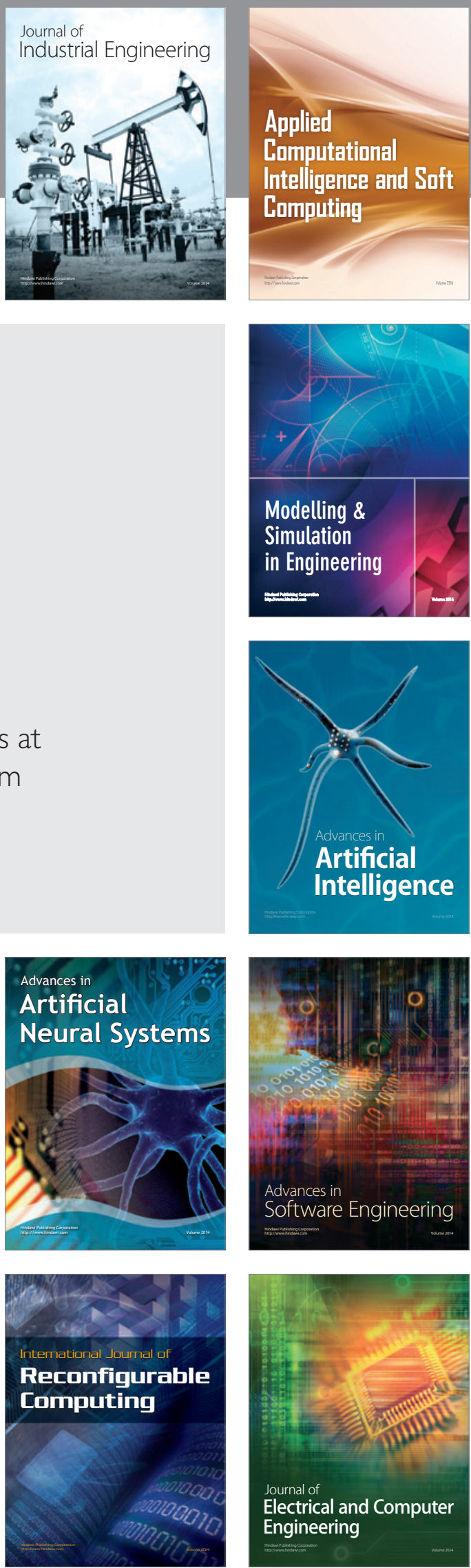